\title{
Pharmacokinetic Parameter Reason Not Done
}

National Cancer Institute

\section{Source}

National Cancer Institute. Pharmacokinetic Parameter Reason Not Done. NCI

Thesaurus. Code C87983.

The explanation given as to why pharmacokinetic parameter was not assessed. 